\title{
Fatores prognósticos da revascularização na fase aguda do infarto agudo do miocárdio
}

\author{
Fabio B. JATENE*, José Carlos NICOLAU*, Alexandre Ciappina HUEB*, \\ Fernando Antibas ATIK*, Luciano M. BARAFIOLE*, Cláudio B. MURTA*, \\ Noedir A. G. STOLF*, Sérgio Almeida de OLIVEIRA*
}

RBCCV 44205-547

\begin{abstract}
Jatene F B, Nicolau J C, Hueb A C, Atik F A, Barafiole L M, Murta C B, Stolf N A G, Oliveira S A - Fatores prognósticos da revascularização na fase aguda do infarto agudo do miocárdio. Rev Bras Cir Cardiovasc 2001; 16(3): 195-202.
\end{abstract}

RESUMO: Objetivo: Determinar os fatores preditores de má evolução nos pacientes submetidos a revascularização do miocárdio (RM) na fase aguda do infarto do miocárdio (IAM).

Casuística e Métodos: No período de março de 1998 a novembro de 1999, 49 pacientes foram submetidos a RM na fase aguda do IAM. Foram excluídos pacientes portadores de complicações mecânicas do IAM e submetidos a procedimentos associados a RM. Os pacientes foram divididos em: Grupo I - 29 casos que não apresentaram complicações decorrentes do IAM e Grupo II - 20 casos com uma ou mais complicações. As complicações consideradas foram: isquemia recorrente (18 pacientes), insuficiência cardíaca congestiva (11), choque cardiogênico (9), hipotensão (7), reinfarto (4), taquicardia ventricular sustentada (4) e fibrilação ventricular (3). Os grupos foram considerados comparáveis em relação às características pré-operatórias, exceto pela idade mais avançada no grupo II. No intuito de identificar os fatores determinantes de pior prognóstico pós-operatório, foram correlacionadas e analisadas as características dos pacientes e as complicações do IAM, estudados pelo teste de variância e análise multivariada.

Resultados: A mortalidade global foi de $6,12 \%$ (3 pacientes), sendo somente no grupo II. A análise multivariada identificou como fatores preditores de mortalidade hospitalar a hipotensão arterial $(p=0,045)$, o choque cardiogênico $(p=0,001)$ e a fibrilação ventricular $(p=0,012)$.

Conclusões: A RM na fase aguda do IAM é um procedimento seguro em pacientes sem complicações, sem mortalidade operatória. A presença de complicações pré-operatórias como choque cardiogênico, fibrilação ventricular e hipotensão são considerados fatores de mau prognóstico nesta condição.

DESCRITORES: Revascularização miorcárdica, métodos. Infarto do miocárdio, cirurgia. Revascularização miocárdica, prognóstico. Revascularização miocárdica, fatores de risco.

\footnotetext{
Trabalho realizado no Instituto do Coração do Hospital das Clínicas da Faculdade de Medicina da Universidade de São Paulo. São Paulo, SP, Brasil. Apresentado ao $27^{\circ}$ Congresso Nacional de Cirurgia Cardíaca. Rio de Janeiro, RJ, 23 a 25 de março, 2000.

* Do Instituto do Coração do Hospital das Clínicas da Faculdade de Medicina da Universidade de São Paulo.

Endereço para correspondência: Fabio B. Jatene. Av. Dr. Enéas de Carvalho Aguiar, 44. São Paulo, SP, Brasil. CEP: 05403-000. Tel.: (11) $3069-5372$. Fax: (11) 3069-5248. e.mail: fabiojatene@incor.usp.br
} 
Jatene F B, Nicolau J C, Hueb A C, Atik F A, Barafiole L M, Murta C B, Stolf N A G, Oliveira S A - Fatores prognósticos da revascularização na fase aguda do infarto agudo do miocárdio. Rev Bras Cir Cardiovasc 2001; 16(3): 195-202.

\section{INTRODUÇÃO}

A indicação de operação de revascularização do miocárdio (RM) precoce, realizada logo após o infarto (IAM), tem sido amplamente discutida com base em observações clínicas, sem que haja consenso sobre os riscos e benefícios deste procedimento, bem como, sobre o intervalo de tempo ideal entre o procedimento e o IAM (1-4).

Durante muito tempo a revascularização precoce foi considerada de risco. Entretanto, mais recentemente, têm sido registrados baixos indíces de morbi-mortalidade em pacientes operados nos primeiros dias ou semanas após o IAM.

Dados sugerem que o tempo é um fator determinante na evolução do paciente infartado. A reperfusão cirúrgica quando realizada nas primeiras 4 a 6 horas resulta em redução da área infartada e preservação da função ventricular esquerda (5). Entretanto, com a grande incorporação dos métodos de reperfusão na fase aguda do IAM, quer sejam farmacológicos ou hemodinâmicos, a revascularização cirúrgica caiu em desuso nesta condição. Ela estaria indicada caso sobrevenha alguma complicação.

O resultado da operação parece estar associado à presença de fatores de risco pré-operatórios específicos ${ }^{(6,7)}$. Fatores como sexo, idade ${ }^{(7)}$, presença de choque cardiogênico (8) e infarto de onda $Q^{(2,6)}$ parecem determinar um mau prognóstico para estes pacientes.

Dentro deste contexto, se insere este estudo que objetiva determinar quais os fatores preditores de má evolução nos pacientes submetidos à RM na fase aguda do IAM.

\section{CASUÍSTICA E MÉTODOS}

No período de Março de 1998 a Novembro de 1999, foram admitidos 318 pacientes com diagnóstico de infarto agudo do miocárdio (IAM) na Unidade Coronariana do Instituto do Coração do Hospital das Clínicas da Faculdade de Medicina da Universidade de São Paulo.

Quarenta e nove $(15,4 \%)$ pacientes foram submetidos a revascularização cirúrgica isolada do miocárdio (RM) na fase aguda do infarto durante a mesma internação. O intervalo médio entre a ocorrência do $\mathrm{IAM}$ e a $\mathrm{RM}$ foi de 12,3 $\pm 6,2$ dias. A presença de complicações mecânicas do IAM ou procedimentos associados à RM foram considerados como critérios de exclusão.

Estes pacientes foram divididos em 2 grupos, de acordo com a ocorrência ou não de complica-
TABELA 1

\begin{tabular}{lcc}
\hline \multicolumn{3}{c}{ CARACTERÍSTICA DOS GRUPOS } \\
\hline CARACTERÍSTICA & GRUPO I & GRUPO II \\
\hline Idade (média + DP) & $57,1 \pm 10,8$ & $64,5 \pm 11,3$ \\
\hline Sexo Masculino & $24(82,7 \%)$ & $15(75 \%)$ \\
Tipo IAM Tipo Q & $21(72,5 \%)$ & $16(80 \%)$ \\
Diabetes Mellitus II & $10(34,5 \%)$ & $7(35 \%)$ \\
IAM prévio & $6(20 \%)$ & $4(20 \%)$ \\
RM prévia & $1(3 \%)$ & $2(10 \%)$ \\
Trombólise Química & $18(62 \%)$ & $5(25 \%)$ \\
Enxertos Utilizados $\left(\mathrm{n}^{\circ}\right)$ & $102(3,5 /$ pac.) & $64(3,2 /$ pac.) \\
Enxertos Arteriais $\left(\mathrm{n}^{\circ}\right)$ & $50(1,72 /$ pac.) & $18(0,9 /$ pac.) \\
Complicações do IAM (\%) & 0 & $100 \%$ \\
\hline
\end{tabular}

ções decorrentes do IAM. Assim os pacientes foram agrupados da seguinte forma:

- Grupo I - 29 pacientes que não apresentavam complicações decorrentes do IAM;

- Grupo II - 20 pacientes que apresentavam uma ou mais complicações decorrentes do IAM.

Estes pacientes foram caracterizados de acordo com idade, sexo, tipo do IAM (IAM Q e IAM não $\mathrm{Q}$ ), presença ou não de diabetes mellitus tipo II (DM), presença ou não de infarto prévio, revascularização prévia do miocárdio e se foram submetidos a trombólise química. Esses dados estão representados na Tabela 1.

Independente da ocorrência ou não de complicação do IAM, a parede mais acometida foi a inferior em $25(51 \%)$ pacientes, seguida da parede anterior em $18(36,8 \%)$ e lateral em $6(12,2 \%)$. A média de enxertos utilizados durante a RM foi de 3,4 enxerto/paciente sendo 68 (1,4 enxerto/paciente) arteriais e 98 (2,0 enxerto/paciente) venosos. A artéria torácica interna esquerda foi utilizada em 43 $(87,7 \%)$ pacientes, a artéria torácica interna direita em $14(28,5 \%)$, a artéria radial em $10(20,4 \%)$ e a artéria gastroepiplóica em 1 (2\%) paciente.

No intuito de identificar quais seriam os fatores determinantes de prognóstico pós-operatório foram, prospectivamente, correlacionadas e analisadas as características dos pacientes e as complicações decorrentes do IAM, no grupo onde elas ocorreram (Grupo II).

Analisou-se a presença de: isquemia recorrente, insuficiência cardíaca congestiva (ICC), choque cardiogênico, hipotensão, reinfarto, taquicardia ventricular sustentada (TVS) e fibrilação ventricular (FV) (Tabela 2). 
Jatene F B, Nicolau J C, Hueb A C, Atik F A, Barafiole L M, Murta C B, Stolf N A G, Oliveira S A - Fatores prognósticos da revascularização na fase aguda do infarto agudo do miocárdio. Rev Bras Cir Cardiovasc 2001; 16(3): 195-202.

Os dados obtidos foram analisados pelo teste de análise de variância e análise multivariada. Foram considerados significativos os valores de $\mathrm{p}<0,05$.

\section{RESULTADOS}

Os dados obtidos foram analisados e correlacionados com a mortalidade.

A mortalidade global foi de $3(6,12 \%)$ pacientes. O Grupo I (29 pacientes) sem complicações decorrentes do IAM não apresentou óbito hospitalar. No Grupo II (20 pacientes) houve 3 (15\%) óbitos.

Analisando as características de ambos os grupos e, comparando os dados do Grupo I em relação ao Grupo II, como: a idade, sexo, tipo do IAM, presença de DM, presença de infarto prévio, revascularização prévia e trombólise química, observou-se diferença estatística significativa nas variáveis: idade com $p=0,017$ (IC $95 \% 1,4$ a 13,3) quando comparou-se o Grupo I com o Grupo II. Não se observou significância estatística da variável idade quando relacionada com a mortalidade. Observouse diferença estatística para a variável: presença ou não de complicações decorrentes do IAM quando relacionada com a mortalidade. Esses dados estão representados na Tabela 3 .

Com relação à análise das complicações decorrentes do IAM, presentes nos pacientes do Grupo II, observou-se que a: hipotensão arterial, o choque cardiogênico e a fibrilação ventricular, quando analisados de forma multivariada estão relacionadas a aumento de mortalidade pós-operatória. Estes dados, bem como a análise das outras complicações, que não foram estatisticamente correlacionadas ao aumento da mortalidade estão representadas na Tabela 4.

\section{COMENTÁRIOS}

Os revolucionários avanços que o tratamento da insuficiência coronária aguda e do IAM têm experimentado nas últimas décadas, com o melhor conhecimento de sua fisiopatologia, levaram a uma redução significativa de seus índices de morbimortalidade.

Os fatores responsáveis por este feito são a utilização precoce e em larga escala dos medicamentos, particularmente dos agentes trombolíticos e dos procedimentos intervencionistas, respeitando o conceito de menor tempo entre o IAM e o tratamento, com maior preservação da função miocárdica e menor mortalidade ${ }^{(5)}$. Em relação à operação, apesar de uma fase inicial controversa, considerouse na década de 70 que a RM deveria ser poster-
TABELA 2

\begin{tabular}{lcc}
\hline \multicolumn{3}{c}{ COMPLICAÇÕES DECORRENTES DO IAM } \\
\hline COMPLICAÇÃo & NÚMERO & $\%$ \\
Isquemia Recorrente & 18 & 90 \\
\hline ICC & 11 & 55 \\
Choque Cardiogênico & 9 & 45 \\
Hipotensão Arterial & 7 & 35 \\
Reinfarto & 4 & 20 \\
\hline Taquicardia Ventricular Sustentada & 4 & 20 \\
Fibrilação Ventricular & 3 & 15 \\
\hline
\end{tabular}

TABELA 3

\begin{tabular}{lc}
\hline \multicolumn{2}{c}{ ANÁLISE ESTATÍSTICA ENTRE OS GRUPOS } \\
\hline CARACTERÍSTICA & $\mathbf{p}$ \\
\hline Idade (média + DP) & 0,017 \\
\hline Sexo & $\mathrm{ns}$ \\
\hline Tipo IAM Q & $\mathrm{ns}$ \\
\hline Diabetes Mellitus II & $\mathrm{ns}$ \\
\hline IAM prévio & $\mathrm{ns}$ \\
\hline RM prévia & $\mathrm{ns}$ \\
\hline Trombólise Química & $\mathrm{ns}$ \\
\hline Enxertos Utilizados $\left(\mathrm{n}^{\circ}\right)$ & $\mathrm{ns}$ \\
\hline Enxertos Arteriais $\left(\mathrm{n}^{\circ}\right)$ & $\mathrm{ns}$ \\
\hline Complicações do IAM & 0,001 \\
\hline
\end{tabular}

TABELA 4

\begin{tabular}{|c|c|c|c|c|}
\hline \multicolumn{5}{|c|}{ ANÁLISE DAS COMPLICAÇÕES DECORRENTES DO IAM } \\
\hline COMPLICAÇÃO & NÚMERO & ÓBITOS & $\%$ & p \\
\hline Isquemia Recorrente & 18 & 3 & 17 & 0,317 \\
\hline ICC & 11 & 2 & 18 & 0,631 \\
\hline $\begin{array}{l}\text { Choque } \\
\text { Cardiogênico }\end{array}$ & 9 & 3 & 33 & 0,001 \\
\hline $\begin{array}{l}\text { Hipotensão } \\
\text { Arterial }\end{array}$ & 7 & 2 & 29 & 0,045 \\
\hline Reinfarto & 4 & 0 & 0 & 0,799 \\
\hline $\begin{array}{l}\text { Taquicardia } \\
\text { Ventricular } \\
\text { Sustentada }\end{array}$ & 4 & 1 & 25 & 0,379 \\
\hline Fibrilação Ventricular & 3 & 2 & 66 & 0,012 \\
\hline
\end{tabular}

gada para acima de 30 dias após a ocorrência do infarto, pelos pobres resultados do mesmo como opção primária (por volta de $20 \%$ de mortalidade) $(2,9)$. Um outro fator que prejudicou a indicação da 
Jatene F B, Nicolau J C, Hueb A C, Atik F A, Barafiole L M, Murta C B, Stolf N A G, Oliveira S A - Fatores prognósticos da revascularização na fase aguda do infarto agudo do miocárdio. Rev Bras Cir Cardiovasc 2001; 16(3): 195-202.

operação na fase aguda foi a alteração de coagulação (10) provocada pelas drogas trombolíticas, levando a um aumento da morbidade cirúrgica.

Entretanto, os resultados da RM da atualidade não podem ser comparados com aqueles obtidos na década de 70 e que se mostravam pouco promissores. Este novo cenário (11) deve-se aos avanços tecnológicos incorporados e ao melhor conhecimento de seus resultados e seleção dos doentes (12), no progresso da circulação extracorpórea, da proteção miocárdica, no maior uso de enxertos arteriais, no uso do balão intra-aórtico ${ }^{(13)}$, da assistência circulatória mecânica (14), no melhor manejo pós-operatório e até na operação sem circulação extracorpórea (15).

Desta forma, a indicação da RM na fase aguda do IAM continua sendo tema controverso, com base em observações clínicas ${ }^{(3,16)}$, sem que haja consenso sobre seus riscos e benefícios, bem como sobre o momento ideal de sua indicação ${ }^{(1-3)}$. Sabese que quando realizada de maneira emergencial, está associada a maior mortalidade, que varia de 4 a $32 \%{ }^{(17-19)}$.

A indicação da RM neste grupo de pacientes passará a ser definida de maneira individualizada, na dependência da caracterização de grupos de risco $(6,7)$. As indicações de RM atuais incluem as complicações mecânicas do IAM, a angina instável, refratária à terapêutica clínica máxima e o insucesso da angioplastia transluminal coronária e dos agentes trombolíticos. Além dessas, são indicações os pacientes que, a despeito de boa evolução clínica pós-IAM, apresentam lesões multiarteriais ou mesmo lesões isoladas de características particulares e que recomendam a indicação cirúrgica.

BRAUNWALD (20) considerou algumas vantagens para a operação, como tratar definitivamente as lesões coronarianas, mesmo as residuais, persistentes após angioplastia ou uso de trombolítico. Isto pode ser facilmente comprovado por estudos que demonstraram que pacientes com angina pós-IAM possuem um risco de até $86 \%$ de apresentarem novo IAM em 1 ano quando tratados clinicamente $(21,22)$; e taxa de mortalidade é de $29 \%$ se o segundo episódio for um IAM transmural (23). Outras vantagens seriam a limitação da extensão do infarto e do remodelamento ventricular que poderia determinar a formação de aneurisma ou rotura ventricular (24). Contudo, há o risco da lesão de reperfusão, com infarto hemorrágico resultante e aumento da perda de contratilidade miocárdica (25).

A mortalidade observada no grupo de pacientes submetidos à revascularização isolada $(4,1 \%)$ em nosso estudo é similar à apresentada por outros autores como HOCHMAN et al. (26) $(4,3 \%)$, HOWARD et al. (27) $(4,3 \%)$ e KAUL et al. (7) (5,9\%). A elevada morbi-mortalidade cirúrgica que pode acompanhar este grupo de pacientes é, via de regra, reflexo da má condição clínica pré-operatória (3), expressa através de fatores preditivos específicos.

Identificamos como fatores de risco significativos pré-operatórios, na análise multivariada, a hipotensão arterial, a fibrilação ventricular e o choque cardiogênico. Este último tem sido apontado por vários autores ${ }^{11,15}$, dentre eles HOCHMAN et al. (26), DONATELLI et al. (8) e KAUL et al. (7) como determinantes de mau prognóstico, sendo por vezes indicativo de insucesso do método de reperfusão (angioplastia primária (27) ou trombolítico).

Outros fatores estudados neste trabalho não obtiveram significância estatística, mas são citados na literatura como importantes. A idade do paciente foi apontada como um fator relacionado a maior mortalidade, conforme apontado por APPLEBAUM et al. (28) e KAUL et al. (7), em pacientes com mais de 70 anos de idade. A idade média neste estudo foi de $60,3 \pm 11,6$ anos, não tendo sido observada associação estatisticamente significativa entre a mortalidade e a idade superior a 65 anos.

O sexo feminino tem sido apontado por alguns autores, dentre eles KAUL et al. (7), como preditor de mortalidade hospitalar após RM. Na nossa experiência, esta correlação não foi constatada talvez pelo reduzido tamanho da amostra. Outros autores como APPLEBAUM et al. (28) e NAUNHEIM et al. (1) também não encontraram associação entre esta variável e a mortalidade imediata.

A constatação do benefício do amplo uso de enxertos arteriais, especialmente da artéria torácica interna, e a preservação do conceito de revascularização completa do miocárdio foi mantida na nossa Instituição e neste grupo de doentes. Desta maneira, o número médio de enxertos utilizados foi de 3,2 enxertos/paciente, sendo usados em média 1,3 enxerto arterial/paciente e 1,9 enxerto venoso/ paciente.

Há uma tendência a considerar pacientes de pior prognóstico para RM nesta fase, aqueles que sofreram IAM de onda $Q$, quando comparados aos que sofreram IAM não-Q. LEE et al. (29), em estudo multicêntrico com 44365 pacientes, encontraram uma maior mortalidade no grupo infartado com onda $Q$ apenas quando foram operados na primeira semana após o IAM. Não observamos associação estatisticamente significante com relação à mortalidade hospitalar entre os grupos infarto $Q$ e infarto não$Q$ por nós avaliados.

Diversos autores têm se preocupado com o tempo ideal da realização do ato cirúrgico $(1,2,4,22)$. 
Jatene F B, Nicolau J C, Hueb A C, Atik F A, Barafiole L M, Murta C B, Stolf N A G, Oliveira S A - Fatores prognósticos da revascularização na fase aguda do infarto agudo do miocárdio. Rev Bras Cir Cardiovasc 2001; 16(3): $195-202$.

A possível alta morbi-mortalidade da RM quando realizada na fase aguda é o principal argumento daqueles que entendem que o procedimento cirúrgico deva ser postergado. Aqueles que tem um pensamento contrário a este, acrediatam que a reperfusão precoce diminuiria o dano ao miocárdio e, portanto, o paciente teria uma evolução mais favorável ${ }^{(5,26)}$.

Este é um dos pontos mais controversos a respeito da RM na fase aguda do IAM. Podem ser identificados dois grupos distintos de pacientes após - IAM, na dependência ou não das complicações, e com evolução pós-operatória distinta, conforme exemplificado neste estudo. Observa-se, portanto, que a cirurgia em pacientes sem complicações tem risco baixo. A média de espera neste grupo, na nossa experiência foi de 12 dias e há uma tendência para que estes pacientes sejam encaminhados para a operação, cada vez mais precocemente. SINTEK et al. (22) concluíram que o intervalo de tempo entre - IAM e a operação não está associado à mortalidade; além disso, não observaram diferenças quanto ao período de permanência hospitalar, índice de acidentes neurológicos, insuficiência renal ou pulmonar. Mesmo no nosso grupo de pacientes com algum grau de complicação, este intervalo de tempo que foi, em média, ao redor de 17 dias, está sendo reduzido. Isto tem importantes desdobramentos, pois fica claro que pacientes sem complicações, com indicação de RM, podem ser operados precocemente, com baixo risco, à semelhança de casos eletivos. Isto cria uma facilidade no manuseio destes pacientes, que via de regra, após o IAM, ficavam aguardando a operação por vários dias, quer seja no hospital, ou ambulatorialmente. Havia, portanto, sempre o inconveniente da espera, quer seja no ambiente hospitalar ou fora dele, com os problemas e os riscos inerentes a cada condição.

\section{CONCLUSÕES}

Pode-se concluir no presente estudo que a revascularização cirúrgica do miocárdio na fase aguda do IAM foi um procedimento seguro em pacientes sem complicações, sem mortalidade operatória. A presença de complicações préoperatórias como choque cardiogênico, fibrilação ventricular e hipotensão é considerada fator de mau prognóstico nesta condição.

Jatene F B, Nicolau J C, Hueb A C, Atik F A, Barafiole L M, Murta C B, Stolf N A G, Oliveira S A - Prognostic factors of myocardial revascularization in acute myocardial infaction. Rev Bras Cir Cardiovasc 2001; 16(3): 195-202.

ABSTRACT: Objectives: To determine the predictors of bad evolution in patients submitted to myocardial revascularization (MR) in the acute myocardial infarction (AMI).

Material and Methods: Between March 1998 and November 1999, 49 patients were submitted to MR in AMI. Patients with mechanical complications of the AMI and those submitted to associated procedures to MR were excluded. The patients were divided into two groups: Group I - 29 cases without AMI related complications and Group II -20 cases with one or more complications. The later ones included persistent ischemia (18 patients), congestive heart failure (11), cardiogenic shock (9), hypotension (7), recurrent AMI (4), sustained ventricular tachycardia (4) and ventricular fibrillation (3). Both groups were considered comparable in terms of preoperative demografics, except for older patients in Group II. In order to determine the prognostic factors for early mortality, the patients profiles and AMI complications were analysed by multivariate and variance tests.

Results: The overall mortality was $6.12 \%$ (3 patients), all of them in Group II. The multivariate analysis identified as predictors of hospital mortality arterial hypotension $(p=0.045)$, cardiogenic shock $(p=0.001)$ and ventricular fibrillation $(\mathrm{p}=0.012)$.

Conclusions: MR in AMI is a safe procedure in patients without preoperative complications, with no deaths. The presence of preoperative complications such as cardiogenic shock, ventricular fibrillation and hypotension were considered predictors of bad evolution in this condition.

DESCRIPTORS: Myocardial revascularization, methods. Myocardial infarction, surgery. Myocardial, revascularization, prognosis. Myocardial revascularization, risk factors. 
Jatene F B, Nicolau J C, Hueb A C, Atik F A, Barafiole L M, Murta C B, Stolf N A G, Oliveira S A - Fatores prognósticos da revascularização na fase aguda do infarto agudo do miocárdio. Rev Bras Cir Cardiovasc 2001; 16(3): 195-202.

\section{REFERÊNCIAS BIBLIOGRÁFICAS}

$1 \quad$ Naunheim KS, Kesler KA, Kanter KR et al. - Coronary artery bypass for recent infarction: predictors of mortality. Circulation 1988; 78 (3 pt 2): I122-8.

2 Braxton JH, Hammond GL, Letsou GV et al. - Optimal timing of coronary artery bypass graft surgery after acute myocardial infarction. Circulation 1995; 92(9 suppl): II66-8.

3 Creswell LL, Moulton MJ, Cox JL, Rosenbloom M Revascularization after acute myocardial infarction. Ann Thorac Surg 1995; 60: 19-26.

4 Deeik RK, Schmitt TM, Ihrig TG, Sugimoto JT Appropriate timing of elective coronary artery bypass graft surgery following acute myocardial infarction. Am J Surg 1998; 176: 581-5.

5 Silverman NA - The surgeon's role in the treatment of acute myocardial infarction. Surg Clin North Am 1985; 65: 527-37.

6 Kennedy JW, Ivey TD, Misbach G et al. - Coronary artery bypass graft surgery early after acute myocardial infarction. Circulation 1989; 79 (6 pt 2): 173-8.

7 Kaul TK, Fields BL, Riggins SL, Dacumos GC, Wyatt DA, Jones CR - Coronary artery bypass grafting within 30 days of an acute myocardial infarction. Ann Thorac Surg 1995; 59: 1169-76.

8 Donatelli F, Benussi S, Triggiani M, Guarracino F, Marchetto G, Grossi A - Surgical treatment for lifethreatening acute myocardial infarction: a prospective protocol. Eur J Cardiothorac Surg 1997; 11: 228-33.

9 Dawson JT, Hall RJ, Hallman GL, Cooley DA - Mortality in patients undergoing coronary artery bypass surgery after myocardial infarction. Am J Cardiol 1974; 33: 483-6.

10 Jatene FB, Jatene MB, Monteiro AC et al. - Revascularização miocárdica após reperfusão as fase aguda do infarto do miocárdio. Arq Bras Cardiol 1991; 56: 295-8.

11 Beyersdorf F, Mitrev Z, Sarai K et al. - Changing patterns of patients undergoing emergency surgical revascularization for acute coronary occlusion: importance of myocardial protection techniques. $J$ Thorac Cardiovasc Surg 1993; 106: 137-48.

12 Bana A, Yadava OP, Ghadiok R, Selot N - Myocardial revascularization after acute myocardial infarction. Int J Cardiol 1999; 69: 209-16.

13 Creswell LL, Rosenbloom M, Cox JL et al. - Intraaortic baloon counterpulsation: patterns of usage and outcome in cardiac surgery patients. Ann Thorac Surg 1992; 54: 11-20.

14 Chen JM, DeRose JJ, Slater JP et al. - Improved survival rates support left ventricular assist device implantation early after myocardial infarction. J Am Coll Cardiol 1999; 33: 1903-8.

15 Mohr R, Moshkovitch Y, Shapira I, Amir G, Hod H, Gurevitch J - Coronary artery bypass without cardiopulmonary bypass for patients with acute myocardial infarction. J Thorac Cardiovasc Surg 1999; 118: $50-6$.

16 Nicolau JC, Ardito RV, Garzon SA et al. - Surgical revascularization after fibrinolysis in acute myocardial infarction: long-term follow-up. J Thorac Cardiovasc Surg 1994; 107: 1454-9.

17 Coleman WS, DeWood MA, Berg Jr. R, Selinger SL, Leonard JJ, Siwek LG - Surgical intervention in acute myocardial infarction: an historical perspective. Semin Thorac Cardiovasc Surg 1995; 7: 176-83.

18 Guyton RA - Aggressive intervention in acute myocardial infarction: a surgeon's perspective. Semin Thorac Cardiovasc Surg 1995; 7: 174-5.

19 Athanasuleas CL, Geer DA, Arciniegas JG et al. - A reappraisal of surgical intervention for acute myocardial infarction. J Thorac Cardiovasc Surg 1987; 93: $405-14$.

20 Braunwald E - Myocardial reperfusion, limitation of infarct size, reduction of left ventricular dysfunction, and improved survival: should the paradigm be expanded? Circulation 1989; 79: 441-4.

21 Brundage BH, Ullyot DJ, Winokur L, Chatterjee K, Ports $\mathrm{TA}$, Turley $\mathrm{K}$ - The role of aortic balloon pumping in post infarction angina: a different perspective. Circulation 1980; 62 (2 pt 2): I119-23.

22 Sintek CF, Pfeffer TA, Khonsari S - Surgical revascularization after acute myocardial infarction: does timing make a difference? J Thorac Cardiovasc Surg 1994; 107: 1317-22.

23 Levine FH, Gold HK, Leinbach RC, Daggett WM, Austen WG, Buckley MJ - Safe early revascularization for continuing ischemia after acute myocardial infarction. Circulation 1979; 60(2 pt 2): 5-9.

24 Weiss JL, Marino N, Shapio EP - Myocardial infarct expansion: recognition, significance and pathology. Am J Cardiol 1991; 68: 35-40D.

25 Roberts CS, Schoen FJ, Kloner RA - Effect of coronary reperfusion on myocardial hemorrhage and infarct healing. Am J Cardiol 1983; 52: 610-4.

26 Hochman JS, Boland J, Sleeper LA et al. - Current spectrum of cardiogenic shock and effect of early revascularization on mortality: results of an International Registry. SHOCK Registry Investigators. Circulation 1995; 91: 873-81.

27 Howard M, Irarrazaval MJ, Corbalan R et al. - Surgical myocardial revascularization during the first 15 days of evolution of acute myocardial infarction. Rev Med Chil 1996; 124: 37-44.

28 Applebaum R, House R, Rademaker A et al. - Coronary artery bypass grafting within thirty days of acute myocardial infarction: early and late results in 406 patients. J Thorac Cardiovasc Surg 1991; 102: 745-52.

29 Lee DC, Oz MC, Weinberg AD, Lin SX, Ting W-Optimal timing of revascularization: transmural versus nontransmural acute myocardial infarction. Ann Thorac Surg 2001; 71: 1197-204. 
Jatene F B, Nicolau J C, Hueb A C, Atik F A, Barafiole L M, Murta C B, Stolf N A G, Oliveira S A - Fatores prognósticos da revascularização na fase aguda do infarto agudo do miocárdio. Rev Bras Cir Cardiovasc 2001; 16(3): $195-202$.

\section{Discussão}

(transcrições de fita gravada)

\author{
DR. LUIZ ALBERTO OLIVEIRA DALLAN \\ São Paulo, SP
}

Agradeço à Comissão Organizadora por esta oportunidade e parabenizo ao Dr. Fabio pela excelente apresentação. Quanto aos resultados que o Dr. Fabio apresentou podemos dividir os pacientes em 2 grupos, os que evoluíram sem complicações decorrentes do infarto, nos quais a mortalidade foi zero, e aqueles que tiveram complicações, sendo a mortalidade de $17 \%$ neste grupo. Vamos mostrar rapidamente a nossa postura frente a estas complicações do infarto. Temos alguns trabalhos publicados exatamente sobre isto, um deles de 1995 sobre comunicação interventricular (CIV) pós-infarto, onde 42 pacientes foram analisados. Neste grupo de pacientes observamos mortalidade global de 38,9\%, mostrando a gravidade da CIV pós-infarto. Entretanto, quando analisamos a condição hemodinâmica do doente, em pacientes em condição hemodinâmica estável a mortalidade foi de $9,5 \%$, saltando para $66,6 \%$ no choque cardiogênico. Dependendo do tempo do choque cardiogênico, 12, 24 ou mais de 24 horas, a mortalidade eleva-se para $25 \%, 70 \%$, podendo chegar perto de $100 \%$. Assim, a primeira mensagem que nós tiramos é que diante de uma complicação mecânica como a CIV, a operação precoce reduz a mortalidade. $O$ mesmo não podemos dizer em relação à insuficiência mitral isquêmica pós-IAM, pois habitualmente temos maior mortalidade associada à intensidade da insuficiência mitral, à baixa fração de ejeção e, também, à presença de choque cardiogênico. Ao contrário da CIV pós-infarto, na insuficiência mitral, nas formas leves e moderadas, procuramos não operar o paciente na fase aguda, protelamos a operação um pouco mais. Quando a forma é severa existe a necessidade de realizar a operação em caráter de urgência. Nas formas leves e moderadas preferimos tratar o paciente clinicamente, a não ser que exista indicação pela coronariopatia associada. Outra complicação possível é a rotura cardíaca, que aparece em 1,05\% dos pacientes. Basicamente conseguimos tratar estes pacientes nas formas sub-agudas, quando se pode acompanhar a evolução desta rotura através de ecocardiograma e tratar precocemente. Para resumir, à luz do que o Dr. Fabio falou e baseados na literatura, estabeleceríamos a seguinte proposta diante do infarto agudo: inicialmente, o paciente é submetido a trombólise e/ou a angioplastia, tendo possibilidade de 3 tipos de evolução. Na presença de complicações mecânicas, como já citamos, o paciente teria indicação de operação de urgência. Não havendo complicação mecânica, há 2 possibi- lidades: ou existe reserva miocárdica (FE > 30\%), ou ela está diminuída ( $\mathrm{FE}<30 \%$ ); neste último caso haveria necessidade de compensação para depois estudar o paciente para uma possível operação. Se houver reserva miocárdica e o paciente evolui assintomático poderia ser programado um cateterismo eletivo com uma eventual operação, ao redor de 14 dias após o IAM. Entretanto, se houver reserva miocárdica e o paciente apresenta sintomas, o cateterismo é antecipado e dependendo da lesão identificada, a operação pode ser programada de eletivamente ou em caráter de urgência. Esta é basicamente a nossa conduta atual e acredito que esteja de acordo com a adotada pelo Dr. Fabio. Queria perguntar ao Dr. Fabio quanto tempo ele considerou como fase aguda, 1 mês, 3 meses após - IAM? Minha segunda pergunta seria prática, à luz de seus resultados e da literatura, que conduta você tomaria frente a um paciente que chega infartado ao pronto socorro? Quando você estudaria este doente e quando indicaria a operação? Mais uma vez, muito obrigado e parabéns.

\section{DR. ALTAMIRO RIBEIRO DIAS \\ São Paulo, SP}

A título de contribuição, gostaria de acrescentar que anos 70 , além do trabalho do grupo de Spokane que o Dr. Fabio citou, a esta época o Prof. Zerbini fez no Hospital das Clínicas uma série grande de revascularizações na fase aguda do infarto do miocárdio, menos de 6 horas após o evento. Infelizmente, não houve estratificação adequada, os resultados foram ruins em alguns pacientes e bons em outros, e esta experiência acabou sendo abandonada. Considerando-se que esta é uma experiência nacional pioneira, acho que poderia haver um registro.

\section{DR. IVO ABRAHÃO NESRALLA Porto Alegre, RS}

Gostaria de perguntar ao Dr. Fabio a respeito da utilização de balão intra-aórtico antes da operação. Tivemos oportunidade em nossa Instituição, no Hospital do Servidor Público Estadual, de utilizar o balão intra-aórtico em 3 pacientes com choque cardiogênico, em aproximadamente 72 horas antes do procedimento cirúrgico. Nossos resultados foram muito satisfatórios o que nos deixou bastante animados em continuar este tipo de indicação, ou seja, colocar o balão intra-aórtico antes do paciente ser submetido a operação, deixar ele melhorar clinicamente e depois indicar a operação em condições melhores. 
Jatene F B, Nicolau J C, Hueb A C, Atik F A, Barafiole L M, Murta C B, Stolf N A G, Oliveira S A - Fatores prognósticos da revascularização na fase aguda do infarto agudo do miocárdio. Rev Bras Cir Cardiovasc 2001; 16(3): 195-202.

\section{DR. ANTÔNIO CARLOS G. PENNA JR. Marília, $S P$}

Em sua experiência a operação foi realizada em média 12 dias após o IAM. Gostaria de saber se você tem experiência numa fase mais aguda, entre 24 e 48 horas?

\section{DR. ANTÔNIO DE PADUA JAZBIK \\ Rio de Janeiro, RJ}

Neste grupo de pacientes, quanto tempo após a administração do trombolítico você realizou a operação?

\section{DR. JATENE}

(Encerrando)

Agradeço aos comentários e vou tentar responder o mais breve possível. Acho que uma das finalidades deste trabalho é tentar mostrar que alguns fatos não são tão importantes quanto se imaginava, tentando desmistificá-los. Consideramos como fase aguda os primeiros 30 dias após o IAM, com grande parte da literatura considera. Entretanto, nossa experiência básica é operar na $1^{\underline{a}}$ ou $2^{\underline{a}}$ semana, o que mostrou que o doente pode ser operado sem nenhum problema, se ele não mostrou nenhuma complicação pós-infarto. A evolução destes pacientes foi absolutamente normal, em 29-30 doentes a mortalidade foi zero, ou seja, é uma cirurgia convencional. Em relação às perguntas do Dr. Dallan, agradeço aos comentários. Com relação ao intervalo de tempo entre o IAM e a operação, consideramos com fase aguda os primeiros 30 dias, embora praticamente nenhum doente ultrapassou 2 semanas. A média de estudo destes doentes foi de 3 dias após o infarto, um tempo relativamente curto se considerarmos os doentes que apresentaram ou não complicação mecânica. O intervalo médio entre a operação e o infarto foi de 11 dias, mas há uma tendência forte de que os pacientes sejam operados cada vez mais cedo. Este doente traz problemas para o hospital, pois supondo que ele evolua bem, fique na UTI por 3 dias, é transferido para o quarto, já tem diagnóstico (cateterismo é realizado em média após 3 dias do IAM) e indicação de tratamento cirúrgico. Este doente fica no quarto mais 3 ou 4 dias, com possibilidade de alta hospitalar, mas irá aguardar a operação por cerca de 2 semanas. Operar após 30 dias do IAM significa postergar a operação por 2 semanas com o doente ocupando o leito e com custos decorrentes ou ainda mandar o doente para casa, correndo risco de a artéria voltar a ocluir ou eventualmente ocluir outra artéria. A idéia é que este grupo de pacientes possa ser operado operado imediatamente. Temos cada vez operado mais precocemente, ao redor de 1 semana, é hoje o prazo médio, considerando-se a última fase da experiência. Agradeço ao Dr. Altamiro pela importante observação; mostrei a experiência do grupo de Spokane porque revelou uma mortalidade muito baixa naquele momento e isto voltou a alertar a literatura para a possibilidade de operar numa fase muito precoce. Quanto à pergunta do Dr. Ivo, é verdade, o balão intra-aórtico foi usado em boa parte dos pacientes operados com complicação. Nos doentes sem complicação mecânica o balão intra-aórtico não foi usado em nenhum caso. Estamos buscando ainda fatores preditivos de mortalidade, estamos verificando possível associação entre o uso de balão e a redução da mortalidade no grupo com complicações. O problema é que são poucos óbitos no geral, ficando difícil correlacionar. Respondendo ao Dr. Penna, operações em 24-48 horas ocorreram, o tempo médio foi de 11 dias, mas tivemos poucos casos operados nesta primeira fase. A Instituição participa de muitos protocolos de pesquisa e, às vezes, o doente toma a droga e não sabemos se foi placebo ou não e após estabilização este doente vai para a operação. Em todos os doentes nós temos nos comportado como se o doente tivesse tomado drogas que interferissem nos fatores de coagulação. Temos feito análise dos fatores de coagulação e se o coagulograma estiver em ordem, o doente é levado à operação. 